Revista de Comunicación y Salud, 2017, Vol. 7, pp. 199-204

Editado por Cátedra de Comunicación y Salud

ISSN: 2174-5323 (impreso) 2173-1675 (electrónico)

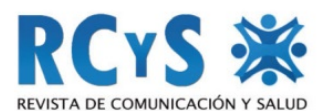

Enviado 16 de septiembre de 2017 Aprobado 11 de noviembre de 2017

\title{
LA MEDICINA TRADICIONAL EN LA POBREZA
}

Traditional medicine in poverty

Cristian José Ávila Martínez ${ }^{1}$

Instituto Mexicano del Seguro Social (IMSS), Zapopan, Jalisco. México

\begin{abstract}
Resumen
La Organización Mundial de la Salud (OMS) ha definido a la "salud" como aquel bienestar físico, mental y social y no solamente la ausencia de enfermedad, La medicina tradicional desde que ha existido junto con la humanidad ha tenido esa visón acerca de la salud, donde mediante su aplicación se sana el cuerpo y el alma para tener un bienestar físico y mental, así como espiritual; creencias desde los antiguos mayas, chinos e indús los cuales decían que cuando se quebranta la salud ha sido por que se ha detenido la relación o conexión del cuerpo y mente hacía con la naturaleza misma. El $80 \%$ de la atencion primaria de la salud a nivel mundial según la OMS es a base de la medicina tradicional y ésta principalmente a nivel rural, ya que el $80 \%$ de los médicos se encuentran en las grandes urbes, mientras que el $20 \%$ restante solo en las áreas rurales, siendo la medicina tradicional una opción a pesar de la no evidencia científica actual para mantener la salud en pacientes con escasos recursos.
\end{abstract}

Palabras clave: Medicina tradicional, salud, poverty.

\begin{abstract}
The World Health Organization (WHO) has defined "health" as that physical, mental and social well-being and not only the absence of disease, traditional medicine since it has existed together with humanity has had that vision about the health, where through its application he heals the body and the soul to have a physical and mental well-being, as well as spiritual; beliefs from the ancient Maya, Chinese and Indus who said that when health is broken, it has been because the relationship or connection of the body and mind with nature itself has been stopped. $80 \%$ of primary health care worldwide according to $\mathrm{WHO}$ is based on traditional medicine and this is mainly at the rural level, since $80 \%$ of doctors are in large cities, while $20 \%$ remaining only in rural areas,
\end{abstract}

\footnotetext{
${ }^{1}$ Autor de contacto: Cristian José Ávila Martínez: dr.cristian_martinez@hotmail.com
} 
traditional medicine being an option despite the current lack of scientific evidence to maintain health in patients with scarce resources.

Keywords: Medicine traditional, health, disease.

\section{Para citar el artículo}

Ávila Martínez, C. J. (2017). La medicina tradicional en la pobreza. Revista de comunicación y salud, Vol. 7, 199-204. Recuperado de http://revistadecomunicacionysalud.org/index.php/rcys/article/view/126

\section{OBJETIVO}

Conocer la importancia que se tiene de la medicina tradicional en las zonas rurales donde coexiste la pobreza, medicina que aún no se tiene una base científica, que no se conocen reacciones adversas y que en este ensayo no se alienta a su uso, sino solo se desea indagar en la importancia y aumento en frecuencia de su uso para mantener la salud a base de creencias o intracultural de generación en generación de un sector de la población tan vulnerable como es la pobreza.

\section{INTRODUCCIÓN}

La pobreza aún coexiste en países en vías de desarrollo a nivel mundial. ${ }^{1}$ La medicina tradicional, llamada así por ser una medicina antigua o local, mientras que aquella denominada complementaria o alternativa es la que es adoptada de otros países de los cuales son de origen, como la acupuntura de China, el uso de la imanenoterapia, inhaloterapia, entre muchas otras más.

Libellus de medicinalibus indorum herbis es el nombre en latín del documento americano más antiguo que hace referencia al empleo de plantas y animales para la elaboración de remedios contra enfermedades. Dentro del traducido Códice de la CruzBadiana también llamado así, obra fundamental para todo aquel estudioso que requiere conocer el mundo de la medicina del centro de Mesoamérica a inicios del siglo XVI, refiere que la medicina tradicional inició con el uso de flora y su fauna en donde existía el uso de 38 animales de los cuales en la actualidad solo 8 son utilizados para la cura de ciertas enfermedades, como lo es la víbora de cascabel, algunos tipos de ave, etc. Estos remedios eran y son utilizados para tratar alrededor de 62 enfermedades: desde pediculosis hasta el auxilio en el trabajo de parto. Las mezclas elaboradas eran frecuentemente hervidas y dadas a beber o bien convertidas en ungüentos, cataplasmas o líquidos que eran untados en las partes afectadas o en el cuerpo completo. También se muestran casos en los cuales el remedio consistía en punzar 
partes dañadas con huesos afilados de animales y también el portar cierto objeto, por ejemplo una roca determinada, para apoyar el tratamiento.

Desde antes de que se desarrollara la medicina de patente, las plantas habían sido utilizadas con fines medicinales; actualmente existe gran conocimiento del uso de las mismas por diferentes grupos de la población mexicana, sobre todo, porque se trata de materia prima de fácil adquisición, sobre todo en comunidades alejadas de las grandes urbes y porque por lo general, son de costo más bajo que los medicamentos alopáticos. Como en países en vías de desarrollo con poblaciones marginadas donde el único recurso para atender la salud por parte de sus pobladores son las hierbas medicinales; por otra parte, existe la creencia de que los preparados herbolarios no tienen riesgo en su consumo y lo que se busca al ingerirlos además de la eficacia terapéutica, es evitar los efectos secundarios que pudieran causar los medicamentos de patente. En algunos casos cabe señalar en la medicina tradicional o la herbolaria principalmente no se utiliza toda una planta, a veces sólo se trata de la raíz, las hojas o las flores, que actualmente al ingerirlas se desconoce a ciencia cierta el beneficio o perjuicio que se tiene al tratar alguna enfermedad en específico.

Algunos estudios sobre el uso de la medicina tradicional como lo es la doradilla, un tipo de helecho que se caracteriza por adquirir una forma de esfera de color dorado en estado seco y volver a reverdecer con la humedad. Esta planta ha sido utilizada desde épocas precortesianas para la obstrucción hepática y la melancolía. De acuerdo a un estudio de campo realizado en el estado de Hidalgo, México se supo que esta planta puede usarse para trastornos del sistema urinario promoviendo la diuresis, y por otra parte, para la dispepsia, irritación del hígado y cálculos biliares.

La denominada escorcionera es una hierba que llega a medir hasta un metro de altura. Su raíz grande de color café es utilizada principalmente por los tarahumaras en México para problemas de la piel, en forma de emplastos que ayudan a la cicatrización de las heridas así como para tratar la artritis misma. Con esa raíz se han realizado pruebas de actividad antimicrobiana que han tenido resultados positivos para hongos dermatofitos, evitando que estos crezcan en la piel.

Con respecto a la medicina complementaria llamase acupuntura, homeopatía, quiropráctica, medicina corporomental, terapias bioelectromagnéticas, sistemas alternativos a la práctica médica, métodos de curación manual, tratamientos farmacológicos y biológicos, dieta y nutrición así como la herbolaria han sido divididos según el Instituto de Medicina Alternativa de los Institutos Nacionales de Salud de los Estados Unidos quien refiere que dice que una de cada tres personas ha usado este tipo de medicina en el último año. 
La medicina alternativa, tradicional o complementaria ha existido, existe y existirá por toda la eternidad, ya que es la medicina a la cual la medicina ortodoxa no ha podido superar en aquellas enfermedades que no ha podido sanar. La medicina tradicional es un escape de principal de esperanza sobre todo en aquellos pacientes con enfermedades crónicas, SIDA, fibromialgia y en general quienes prefieren esta medicina ya sea por razones principalmente económicas, culturales, sociales o porque es la que simplemente conocen como lo es en poblaciones que cohabitan de la mano con la pobreza, y no por ello el medico alópata debería de reprimir, de hecho han existido encuestas en Estados Unidos que refieren que médicos alópatas han usado en $45 \%$ la medicina alternativa.

La medicina ortodoxa o tradicional es y será a libre albedrio del paciente en particular, aunque es claro aclarar y recalar a todo paciente que no deje de tomar algún tratamiento en específico de tipo alópata para mantener estable cualquier enfermedad y está en su decisión el usar además la medicina tradicional, principalmente debe de existir la empatía con el médico y reforzar la relación médico paciente, para en conjunto determinar la preferencia del paciente a la mejor alternativa posible y conocer que tipo de medicina tradicional usa y conocer en lo posible sinergismo o antagonismo e inclusive reacciones adversas tras su uso en conjunto.

Es muy importante también tomar en cuenta que en muchas ocasiones el dinero no es el suficiente como para tener un servicio de salud optimo, en principio porque sus escasos trabajos laborales no son bien renumerados y en segunda no son cubiertos por la mayoría de los servicios de salud gubernamentales. Por lo anterior algo verdaderamente importante es entonces tomar en cuenta el nivel socioeconómico de los pacientes aunque en aquellos usuarios de la medicina tradicional lo más común sea observarlo en aquellos con escasos recursos.

Una gran parte de la población mundial vive en pobreza o en extrema pobreza, donde la salud no es prioridad para ellos sino la alimentación o el cobijo bajo un techo de cartón, por extremar la pobreza que aún se vive en ciertas ciudades, y no les queda más remedio que utilizar lo más práctico, lo que hay a sus alrededores, en el campo, el uso de algunas sustancia o partes de animales al hablarse de la medicina tradicional no se diga la complementaria como loa acupuntura, inhlaloterapia entre otras. Sino lo más práctico y fácil de obtener que por cuestiones culturales que se ha transmitido de generación en generación como lo que está escrito en el antiguo códice de la medicina tradicional de México, hasta lo utilizado hoy en día, que hasta existen tianguis o mercados especializados en esta medicina, donde se vende de acuerdo a la oferta y la demanda determinado producto con finalidad de curar hasta lo inimaginable 
científicamente como lo es un cancer, VIH o SIDA entre otras. La medicina tradicional no debe de pelear en las decisiones de los médicos, pero se debe de advertir a los pacientes cuando la usen en conjunto con la medicina alópata que no se conocen científicamente las reacciones adversas que se puedan tener al utilizar cierta planta o podría poner en riesgo o agravar la salud de nuestros pacientes.

\section{CONCLUSIÓN}

Es importante entonces conocer qué es lo que están utilizando nuestros pacientes, orientarlos en su tratamiento alopático y no negar sin antes establecer medidas preventivas que contribuyan a un mal más que a un beneficio con respecto a la medicina tradicional.

El paciente tiene el derecho de elegir , la mejor terapia que para él le satisfaga, pues está claro que muchas enfermedades no son curables dentro de la ciencia sino solo tratables, por lo tanto siempre la medicina tradicional convergerá a un lado de la medicina científica, el ser humano por igual coexistirá con su cultura de origen y en lo que para ellos han observado resultados y beneficio hacia su enfermedad situación que es aceptable al hablar de que la salud no es solo tratar de curar o mejorar ese estado de determinada enfermedad sino también el integrar el ámbito social, psicológico, bienestar mental, de manera en conjunto con su enfermedades para tener una salud completa sin importar con que medio se llevó a su fin.

\section{BIBLIOGRAFÍA}

CONEVAL. (2016). El desarrollo social en México a partir de la medición de pobreza. México: CONEVAL

Aedo F. J., Granados J.C. (2000). La medicina complementaria en el mundo. Medigraphic, 12, 91-99.

Gómez G, Reyes SR, Teutli C, Valadez R. (2007). La medicina tradicional prehispánica, vertebrados terrestres y productos medicinales de tres mercados del valle de México. Etnobiología 5, 86-98.

Gómez G, James J. (2007). La medicina tradicional y el problema de la salud: inicio de un recorrido en el estudio de la salud mental. Revista científica Guillermo de Ockham. 2007, 5(1), 13-28.

Pérez I. (2013). Medicina tradicional, potencial y riesgos. México: UNAM.

Rojas F, Silva LC, Sansó F, Alonso P. (2013). El debate sobre la medicina natural y tradicional y sus implicaciones para la salud pública. Revista Cubana de salud pública. 39(1), 107-123. 
La medicina tradicional en la pobreza

Schiwa A. En defensa de una medicina natural y tradicional avalada. Revista Cubana de salud pública, 39(4), 623-626.

Vides A, Álvarez A. (2013). La medicina tradicional como un modelo de atencion integral en salud. Revista del valle de Guatemala, 58-60. 